\title{
Isolation of the mRNA-capping enzyme and ferric-reductase-related genes from Candida albicans
}

\author{
Toshiko Yamada-Okabe, ${ }^{1}$ Osamu Shimmi, ${ }^{2}$ Rikuo Doi, ${ }^{1}$ \\ Kiyohisa Mizumoto, ${ }^{3}$ Mikio Arisawa ${ }^{2}$ and Hisafumi Yamada-Okabe ${ }^{2}$
}

Author for correspondence: Hisafumi Yamada-Okabe. Tel: +81467472213 . Fax : +81467465320. e-mail : hisafumi.okabe $(\bar{a})$ roche.com

1 Department of Hygiene, School of Medicine, Yokohama City University, Japan

2 Department of Mycology, Nippon Roche Research Center, 200 Kajiwara, Kamakura, Kanagawa 247, Japan

3 Department of Physiological Chemistry, School of Pharmaceutical Science, Kitasato University, Japan
The mRNA-capping enzyme (mRNA 5'-guanylyltransferase) gene was cloned from a Candida albicans genomic DNA fibrary by functional complementation of a Saccharomyces cerevisiae ceg1 $1 \Delta$ null mutation. This gene, referred to as CGT1 (C. albicans guanylyltransferase 1), can encode a $52 \mathrm{kDa}$ protein that is highly homologous to S. cerevisiae Ceg1p. CGTI in a single-copy plasmid complemented the lethality of the $S$. cerevisiae ceg1 $\Delta$ null mutation and, like S. cerevisiae Ceg1p, bacterially expressed Cgt1p was able to form a stable complex with the GMP moiety of GTP and to synthesize the cap structure in vitro, demonstrating that CGT1 is the $C$. albicans mRNA 5'-guanylyltransferase gene. CGT1 seemed to exist as a single copy in the $C$. albicans genome and was actively transcribed into mRNA. Another ORF was found in an opposite strand very close to the CGI1 locus. This gene shared significant sequence homology with S. cerevisiae FRE1, the gene encoding ferric reductase, and therefore was designated CFL1 ( $C$. albicans ferric-reductase-like gene 1). Despite its sequence homology with S. cerevisiae FRE1, CFL1 MRNA was not induced by iron deprivation, and CFL1 did not complement the slow growth of a S. cerevisiae fre $1 \Delta$ null mutant in the absence of iron, suggesting that $C F L 1$ is functionally distinct from S. cerevisiae FRET.

Keywords: mRNA-capping enzyme, guanylyltransferase, Candida albicans, stable GMP-enzyme complex, fertic reductase

\section{INTRODUCTION}

Almost all eukaryotic mRNAs are capped at their 5'termini (Shatkin, 1976). Capping is tequired for the efficient processing (Edery \& Sonenberg, 1985; Inoue $e t$ al., 1989; Konarska et al., 1984; Krainer et al., 1984; Patzelt et al., 1987; Ohno et al., 1987), stabilization (Furuichi et al., 1977; Shimotohno et al., 1977; Murthy et al., 1991), nuclear export (Hamm \& Mattaj, 1990) and translation of mRNA (reviewed by Filipowicz, 1978; Shatkin, 1985). Capping occurs at an early step during transcription and is catalysed by the capping enzyme. At least three enzymic activities are required for the synthesis of the cap structure: (i) removal of the $\gamma$-phosphoryl

Abbreviations: 5-FOA, 5-fluoroorotic acid, GST, glutathione S-transferase.

The DDBJ accession numbers for the sequences reported in this paper are D83180 for CGT1 and D83181 for CFL1. group of 5'-termini of mRNA by mRNA 5'triphosphatase to produce diphosphate-end RNA; (ii) transfer of the GMP moiety of GTP to the resulting diphosphate end of RNA by mRNA 5'-guanylyltransferase; and (iii) methylation of position 7 of newly produced 5 -terminal guanosine by mRNA-cap methyltransferase. Among these three enzymes, mRNA 5'guanylyltransferase has a unique feature: it covalently binds to the GMP moiety of GTP and forms a stable enzyme-GMP complex (for reviews see Mizumoto \& Kaziro, 1987; Shuman, 1995).

In higher eukaryotes, both mRNA 5'-guanylyltransferase and mRNA $5^{\prime}$-triphosphatase activities are present in a single polypeptide (Yagi et al., 1983), whereas the yeast capping enzyme consists of two subunits: the $52 \mathrm{kDa} \alpha$ subunit is responsible for mRNA $5^{\prime}$-guanylyltransferase activity and mRNA $5^{\prime}$-triphosphatase activity is intrinsic to the $80 \mathrm{kDa} \beta$ subunit (Itoh et al., 1987). Using the antibody raised against purified yeast capping enzyme, the 
CEG1 gene was cloned from a Sacharomyces cerevisiae genomic DNA library and was shown to encode a $52 \mathrm{kDa}$ $\alpha$ subunit (Shibagaki et al., 1992). In addition, distuption of $C E G 1$ was lethal, indicating that $C E G 1$ is essential for vegetative growth of $S$. cerevisiae (Shibagaki et al., 1992). Mutational analyses of the CEG1 gene revealed that $\mathrm{Lys}_{70}$ is important for the covalent binding to GMP; amino acid substitution of $\mathrm{Lys}_{\mathbf{7 0}}$ results in the complete loss of enzyme activity and in the death of $S$. cerevisiae cells (Fresco \& Buratowski, 1994; Schwer \& Shuman, 1994). Furthermore, the amino acid sequence Lys ${ }_{70}$-Thr-AspGly of Ceg1p is highly conserved in mRNA $5^{\prime}$-guanylyltransferases from Schizosaccharomyces pombe and several viruses (Niles t al., 1986; Pena et al., 1993; Seliger $\ell$ al., 1987; Shuman et al., 1994; Upton et al., 1991), indicating that Lys $_{70}$ is located in the active site of the enzyme.

Candida albicans is a dimorphic fungus that commonly causes infection. It grows as a diploid and does not have a sexual cycle (Scherer \& Magee, 1990). Upon stimuli such as serum addition or temperature shift, $C$. albicans cells undergo a morphogenetic transition from the yeast to the hyphal form. This morphogenetic transition seems to be somehow associated with virulence because clinical isolates are more efficiently converted into the hyphal form in vitro (Odds, 1987).

Several azole compounds such as fluconazole and itraconazole have been developed and used for antiCandida chemotherapy. However, a recent increase in azole-resistant $C$. albicans requires the development of a new type of compound having a different mode of action. Capping enzyme seems to be a feasible antifungal target because $m R N A$ capping is essential, even for fungal cells, and the structure of fungal capping enzyme differs from that of the mammalian counterpart (e.g. subunit vs single polypeptide) as described above.

In an attempt to search for a novel antifungal target, we isolated the mRNA-capping enzyme gene from $C$. albicans. The cloned gene shared significant sequence homology with the mRNA $5^{\prime}$-guanylyltransferase genes of $S$. cerevisiae (Shibagaki et al., 1992) and Scbiz. pombe (Shuman et al., 1994), and the protein expressed in bacterial cells was fully active as mRNA $5^{\prime}$-guanylyltransferase.

\section{METHODS}

Generation of ceg $1 \Delta$ and fre $1 \Delta$ null mutant strains. In order

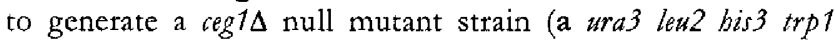
ceg1 $1:$ :LEU2 CEG1-URA3), the entire S. cerevisiae CEG1 ORF that was amplified by PCR using primers possessing a $S \mathrm{maI}$ cleavage site was subcloned at the $S \mathrm{maI}$ site of $\mathrm{pUC18}$, and LEU2 was ligated at a unique EcoRI site of CEG1 in an opposite transcriptional orientation to CEG1. S, rerevisiae diploid RAY3A-D (a/ $\alpha$ ura $3 /$ ura 3 leu2/leu 2 bis $3 /$ bis $3 \operatorname{trp} 1 / \operatorname{trp} 1)$ was then transformed with the resulting CEG1-LEU2 chimeric gene, and several Leu ${ }^{+}$transformants were selected. After confirming the correct integration of the CEG1-LEU2 gene at the CEG1 locus by Southern blotting, the cells were further transformed with YEp24 (Botstein et al., 1979) carrying intact $C E G 1$, and dissected for tetrads. Thus, the resulting ceg1 $1 \Delta$ cells could survive in the absence of 5-fluoroorotic acid (5-FOA) but died when it was present. A S. cerevisiae fre $1 \Delta$ null mutant strain (a $\operatorname{ura} 3$ leu2 bis 3 trp 1 fre $1 \Delta:$ : LEU2) was also obtained by singlestep gene disruption. The entire $S$. cerevisiae FRE1 ORF, which was also amplified by PCR using primers possessing a $S \mathrm{maI}$ cleavage site, was subcloned at the $S \mathrm{maI}$ site of $\mathrm{pLC19}$, and a $2.4 \mathrm{~kb} X b o \mathrm{I}-X b_{0} \mathrm{I}$ fragment of FRE1 was replaced by LEU2, which was inserted in an opposite orientation to FRE1 transcription. S, cerevisiae RAY3A-D cells were then transformed with the resulting FRE1-LEU2 chimeric gene, selected for leucine auxotrophy, and dissected for tetrads. Integration of the disrupted copy of FRE1 was also confirmed by Southern blotting. Primers used for PCR were ${ }^{5}$ AATTACCCGGGAATGG'T'T"TAGCAATG ${ }^{\prime \prime}$ and ${ }^{5}$ TCGTATCCCGGGCTTAGCCTAATCCGA $^{3^{\prime}}$ for CEG1, and ${ }^{5}$ GGATCCCCGGGAATTCATATGGTTAGAACCCGTGTATTATTCTGC ${ }^{3}$ and ${ }^{5}$ GTACCGAGCTCCCCGGGTTACCATGTAAAACTTTCT'TCT'TCTAGT ${ }^{3}$ for FRE1.

Construction of a genomic DNA library and screening for the C. albicans capping enzyme gene. Genomic DNA was extracted from $C$. albicans strain IFO 1060 as described by Lundblad (1989) and partially digested with Sax3AI. The resulting Sau3AI-digested DNA fragments, whose sizes were between 5 and $20 \mathrm{~kb}$, were separated by agarose gel electrophoresis, isolated and ligated at the BamHI cleavage site of YEp24T in which UR $A 3$ (Botstein et al., 1979) was replaced with TRP1 (Struhl et al., 1979). Then, ceg1 1 null mutant cells were transformed with a $C$. albicans genomic DNA library by the lithium acetate method (Ito et al., 1983), selected for tryptophan auxotrophy, and further selected by 5 -FOA resistance (Lundblad, 1989). Nine out of $3 \times 10^{4} \mathrm{Trp}^{+}$transformants were found to grow in the presence of 5-FOA, and plasmid DNA was recovered from the cells grown on the plates containing 5-FOA as described by Lundblad (1989). The inserted DNA fragments were excised by digesting the recovered plasmids with vatious restriction endonucleases and subcloned into $\mathrm{YEp} 24 \mathrm{~T}$ to test for 5-FOA resistance. The DNA fragment that allowed ceg1 $1 \Delta$ null mutant cells to grow in the presence of 5-FOA was digested with several restriction endonucleases, further subcloned in the pUC19 vector, and sequenced using a Sequenase Version $2.0 \mathrm{kit}$ (USB) and $\left[\alpha_{-}{ }^{35} \mathrm{~S}\right] \mathrm{dCTP}$ (NEN).

Southern and Northern blotting. Genomic DNA was extracted from $C$. albicans spheroplasts made by treating the cells with zymolyase and $0.1 \%$ SDS (Lundblad, 1989). After cell debris was removed by centrifugation, DNA was precipitated with ethanol, treated with pancreatic RNase and digested with the indicated endonucleases. For extracting RNA, $C$, albicans cells were cultured in minimum defined (MD) medium in the presence or absence of $2 \mathrm{mM} \mathrm{FeCl}_{3}$ (Dancis et al., 1990) until the $\mathrm{OD}_{600}$ reached $1 \cdot 0$. RNA was then extracted by lysing the cells with glass beads in the presence of SDS, followed by phenol extraction and ethanol precipitation (Kasahara et al., 1994). Poly $(\mathrm{A})^{+}$RNA was purified from total RNA with Oligotex$(\mathrm{dT})_{30}$ as described by Kuribayashi-Ohta et al. (1993). Endonuclease-digested DNA and poly $(\mathrm{A})^{+}$RNA were fractionated by agarose gel electrophoresis, transferred to nylon membranes, hybridized with radiolabelled probes and visualized by autoradiography (Sambrook et al., 1989). The probes used for blotting were the entire PCR-amplified ORFs of CGT1 and CFL 1. Radiolabelling of DNA probes was carried out by the random ptiming method with $\left[\alpha{ }^{32} \mathrm{P}\right] \mathrm{dCTP}$ (Sambrook et al, 1989). Hybridization and washing of the filters were carried out under stringent conditions $[5 \times \mathrm{SSC}(1 \times \mathrm{SSC}$ is $0.15 \mathrm{M} \mathrm{NaCl}$, $0.015 \mathrm{M}$ sodium citrate), $1 \times$ Denhardt's solution $(0.2 \mathrm{~g}$ Ficoll, $0.2 \mathrm{~g}$ polyvinylpyrrolidone, $0.2 \mathrm{~g} \mathrm{BSA} ; \mathrm{l}^{-1}$ ), $20 \mathrm{mM}$ sodium phosphate buffer ( $\mathrm{pH} 6.5$ ), $0.1 \%$ SDS and $50 \%$ formamide at $42{ }^{\circ} \mathrm{C}$ for hybridization; $0.1 \times$ SSC and $0.1 \%$ SDS at $60^{\circ} \mathrm{C}$ for washing) (Sambrook et al, 1989). 


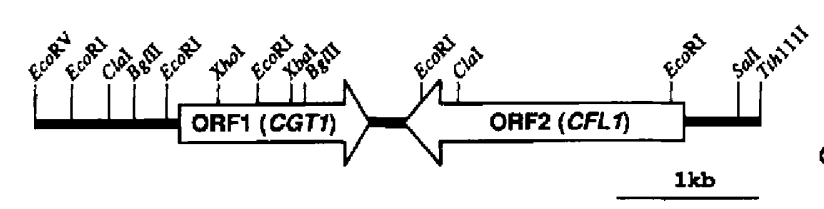

$\stackrel{-1}{-}$

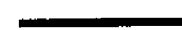

-
Complementation of ceg1 $1 \triangle:$ LEU2

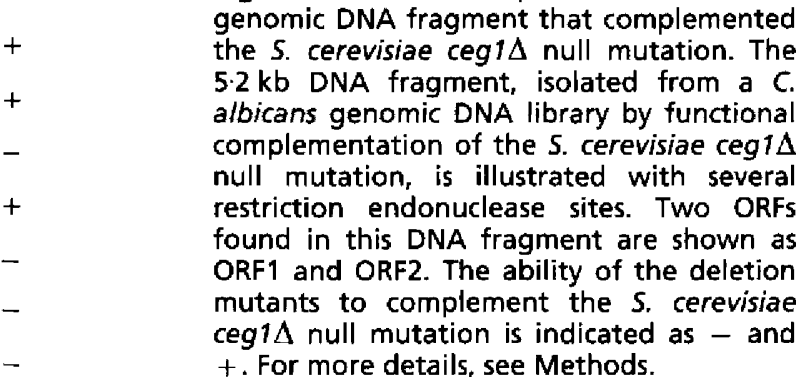

Expression of S. cerevisiae and $\mathbf{C}$. albicans capping enzymes in Escherichia coli. The entire S. cerevisiae CEG1 and C. albicans CGT1 ORFs were amplified by PCR and ligated at the $S \mathrm{maI}$ cleavage site of $\mathrm{pGEX}-2 \mathrm{~T}$, which would result in the expression of Ceg1p and Cgt1p as fusion proteins with glutathione $S$ transferase (GST) in bacterial cells (Smith \& Johnson, 1988). Primers used for amplifying $C E G 1$ have already been described in this section, and those for CGT1 were ${ }^{5} \mathrm{GGATCCCCG}$ GGAAT'TCTTATGA'TCAA'T'TAGAAGAAAGAGAAA TT $^{3}$ and ${ }^{5}$ GTACCGAGC'TCCCCGGGTTATTCGTCGTCACTATCTTCATAAGTG ${ }^{3}$. E. coli XL-1 Blue cells were transformed with the above plasmids and were induced to express $\mathrm{Ceg} 1 \mathrm{p}$ or Cgt1p with $1 \mathrm{mM}$ IPTG at $37^{\circ} \mathrm{C}$ for $4 \mathrm{~h}$. Bacterial cells were then lysed by sonication in buffer $A$ containing $20 \mathrm{mM}$ Tris $/ \mathrm{HCl}$ (pH 7.5), $0.5 \mathrm{mM}$ EDTA, $50 \mathrm{mM} \mathrm{NaCl}$, $10 \mathrm{mM} \beta$-mercaptoethanol, $10 \%$ (v/v) glycerol, $0.05 \%(\mathrm{v} / \mathrm{v})$ Nonidet P40 and $1 \mathrm{mM}$ PMSF (Itoh et al, , 1987). After removing cell debris by centrifugation, GST-Ceg1p and GST - Cgt1p were purified by glutathione-Sepharose CL-4B column chromatography as described by Smith \& Johnson (1988). The purified fusion proteins were dialysed against buffer $A$, assayed for enzyme-GMP complex formation in a standard reaction mixture containing $20 \mathrm{mM}$ Tris/ $\mathrm{HCl}(\mathrm{pH} \mathrm{7.5}), 3 \mathrm{mM} \mathrm{MgCl}, 10 \mathrm{mM}$ dithiothreitol, $0.4 \mathrm{mg}$ bovine serum albumin $\mathrm{ml}^{-1}, 2 \mathrm{mg}$ inorganic pyrophosphatase $\mathrm{ml}^{-1}, 20 \%(\mathrm{v} / \mathrm{v})$ glycerol, $1 \mathrm{mM}$ PMSF and $1 \mathrm{mM}\left[\alpha^{-32} \mathrm{P}\right] \mathrm{G} T \mathrm{TP}$ (specific activity $1 \times 10^{5}$ c.p.m. pmol ${ }^{-1}$ ) at $30^{\circ} \mathrm{C}$ for $10 \mathrm{~min}$ (Itoh et al., 1984), analysed by SDS-PAGE and visualized by autoradiography. In vitro cap formation was assayed according to the method of Mizumoto \& Lipmann (1979) in a $25 \mu \mathrm{l}$ reaction mixture containing $10 \mathrm{mM}$ 'Tris $/ \mathrm{HCl}$ ( $\mathrm{pH} \mathrm{7.5),} 3 \mathrm{mM} \mathrm{MgCl}, 1 \mathrm{mM}$ dithiothreitol, $0.1 \mathrm{mM}$ EDTA, $20 \%(\mathrm{v} / \mathrm{v})$ glycerol, $2 \cdot 4 \mu \mathrm{M}\left[\alpha_{-}{ }^{32} \mathrm{P}\right] \mathrm{GTP}\left(400 \mathrm{Ci} \mathrm{mmol}^{-1}\right.$; $14.8 \mathrm{TBq} \mathrm{mmol}^{-1}$ ), $50 \mathrm{pmol} \mathrm{ppG}$-terminated RNA and $1 \mu \mathrm{g}$ GST-Ceg1p or GST Cgt1p protein at $30^{\circ} \mathrm{C}$ for $60 \mathrm{~min}$. After digesting the RNA with nuclease $\mathrm{P} 1$ and calf intestine alkaline phosphatase, the cap structure was identified by paper electrophoresis on DE81 paper and autoradiography.

Complementation of $\operatorname{ceg} 1 \Delta$ and fre $1 \Delta$ null mutants by CGT1 and CFL1. A $2.5 \mathrm{~kb} \mathrm{ClaI-ClaI} \mathrm{fragment} \mathrm{of} \mathrm{CGT1} \mathrm{and} \mathrm{a} 3.7 \mathrm{~kb}$ $S_{a l}$-Xhol fragment of CFL1 were subcloned in YEp24T or pRS414 and transfected into the ceg $1 \Delta$ null mutant or fre $1 \Delta$ null mutant strains, respectively. The ability of CGT1 or CFL.1 to complement the ceg $1 \Delta$ null mutation or the fre $1 \Delta$ null mutation were examined by culturing the above transfectants on agar plates containing 5-FOA (for CGT1) or on MD medium lacking iron (for CFL1) (Dancis et al., 1990), respectively.

\section{RESULTS}

\section{Cloning of the $C$. albicans mRNA-capping enzyme gene}

We wanted to identify the mRNA-capping enzyme gene in $C$. albicans, one of the pathogenic fungi that causes human mycosis. Since we failed to detect a clear signal by Southern blotting of $C$. albicans genomic DNA using different regions of $S$. cerevisiae CEG1 as probes, the approach we chose was functional complementation of the $S$. cerevisiae ceg1D null mutation. Since CEG1 is an essential gene in S. cerevisiae (Shibagaki et al., 1992), we generated an $S$. cerevisiae strain in which endogenous CEG1 was disrupted but in which intact CEG1 was retained in YEp24 that harboured $U R A 3$ as a selectable marker. This strain, designated $c e g 1 \Delta$, grew in the absence of 5-FOA but died when it was present as a consequence of the elimination of the UR $A 3$-containing plasmid.

ceg1A cells were then transformed with a $C$. albicans genomic DNA library and selected by tryptophan auxotrophy and 5-FOA resistance. Nine out of $3 \times 10^{4}$ $\operatorname{Trp}^{+}$transformants were found to grow in the presence of 5-FOA, and all plasmids recovered from these 5-FOAresistant clones reproducibly conferred resistance to 5 FOA when transfected into ceg1 $1 \Delta$ cells. Restriction endonuclease digestion of the recovered plasmids revealed that all clones carried the same part of the $C$. albicans genomic DNA, while the size of the inserted DNA fragment was different in each clone. One clone carrying the shortest genomic DNA fragment was further digested with restriction endonucleases to make deletions, and a

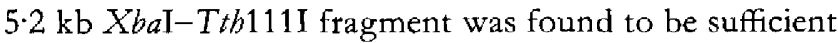
to complement the lethality of the ceg1 $1 \Delta$ null mutant.

Sequencing of the cloned $C$. albicans genomic DNA fragment revealed that it contained two potential ORFs, designated ORF1 and ORF2 (Fig. 1). ORF1 could encode a protein with a molecular mass of $52 \mathrm{kDa}$ that is highly homologous to mRNA $5^{\prime}$-guanylyltransferases of $S$. cerevisiae (Ceg1p) (Shibagaki et al., 1992) and Sobiz. pombe (Pce1p) (Shuman et al., 1994) (39.3\% identity and 76.5\% homology with Ceg1p, and 38.2\% identity and $75.9 \%$ homology with Pcelp, in the entire protein) (Figs $2 a$ and 
(a)

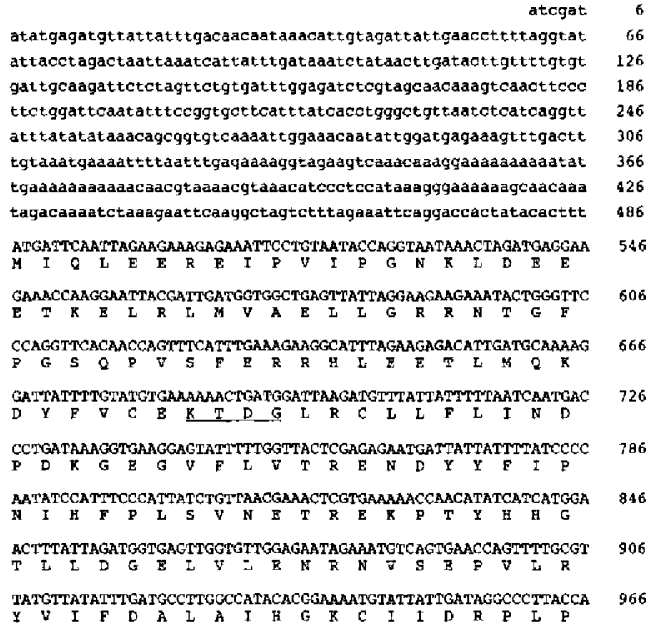

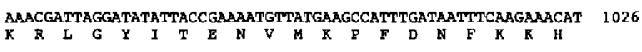

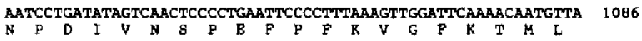
ACTRCTTACCATECTGATGATGTTTTGGGTAGGATGGaATAATTJTTTCATGCTTCAGAT 1146 GgaTTAATTTATACTTGTGCCGAAaCACCTTATGTATTTGGAACTGaCCAAACATTCCTA 1206

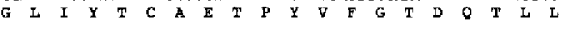
$\underset{K}{\text { AAATGGAAACCCOCTGAAGAGACACTGTTGATPTPCAATTAGAGTTTGTTPTCAATGAA }} 1266$ GTGCAGGATCCAGACTTGGACGAAGGGGCCCAACATCAACTTATCTAGACTACGATGCT 2326 AACCCGAATTTAARAAAATTGAGGGTATGGCAAGGTAGCAATGTACATACTGATTTTGCC 1386

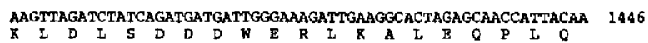

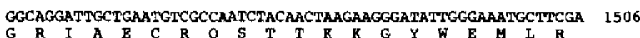

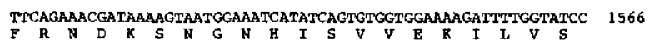
F N D $x$ W ATCAAGCATGGTGTCARAGAGAAAGA GGTCATTGAGTGGTGTCCCAAAATATCAAGGGCI TGGAAGAaGAGAGAGACCATAGAAGACACAAGCATITCAATCGAGTTCCTCGTCCAGCT 1686

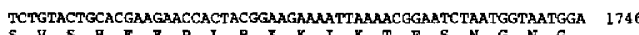

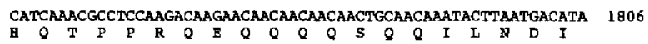
CCCACTTATGARGATAGTGACGACGAATARattgaataagåazcaaaaacaataaaaata 1866 gagtttgattctttctcggtttctgggaaaagaatgacagcatgaatatatttaaataag 1926 aaaaatttaagttgtgtatasatttgtattactagt.atcaataatatataaaggeaatct 1986 aaaataaaaaaaataagataaagtaaagagagaagacttatttacagttgtttttgtttt 2046 caaatcttgtatcact gattatgcccatgretgtaattgttegtgatattccactctgt 2106 getttgatacattcaaatectgggtgactgcaaatctcaactcatcaaceattgcagggt 216.6 gtccacatgtgacaaatccaattgcaccarcagcttgttraacctcrtgttcaacttgcg 2226 tagaaatgtctggacgeccttcaatgaattcaacatgagataacecttgcttaatattag 2286 aaatgagtgaatattgagatgattetactgagtcettttcgtcggatttcttrtcaaaac 2346 taacategtgttcaaagcattctaatcctgagcaatcttgăggttgagtaacataa atgg 2406 tactttgcacgtttgtttttttcaagtattccaattcctcagtgaaccaacttaakgatt 2466 tccagtgtcttataatccaateaacttgatcgat (b)

gtegacgctetceettatgegactcetgcattaggaagca

geccagtagtaggttgaggecgttgagcacegecgccgcaaggaatggtgcatgcaagga gatygegeccaacagteccceygccacggggcetgccacestacceacggocgaaacasg cgctcatgagccogaagtggegagecegatcttocceateggtgatgteggcgata =agg egccageascegcacetgrggegoeggtgatgceggecacgatgogtocggegtagagga tcaactaattggaattgttattgtaaaacggatgctçggtttggaacaatctcagattgt ttagtecgaggtttcaataacaacacaaatatcattagtbaat tracagaatoctgtaat

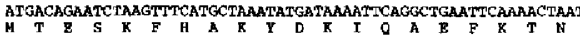
GoTACTGAATATGCTAAGATGACTACCAAGTCATCCAGTGGTAGTAMGACTrCTACTAGT OCAGGTAaATCETCCAAGTCAaCAGGTCTGTCCAATCCCTCGAaGTCGTECACAAATECT 580

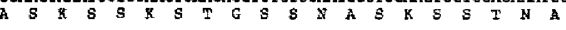

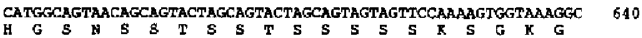
AaTAGCGGTACATCCACAACAGAaACTATAACCACTCCATTGTTAATTGATTATAMAAAA 700 TTCACTCCTTACAAGGATGCTTACCAAATGTCTAATAATAaATTCAATCTPTCAATAaAC 760 F T P Y K K D A Y TACGGTTCAGGGTTACTTGGTTATTGGGCAGGGATTPTACCGATTGCAATTTPTCCTAAC 820 ATGATTAAAAAARTGTTCCCTAGTTTGACAAATAATTTATCTGGTTCGATTTCCAACITA BBO

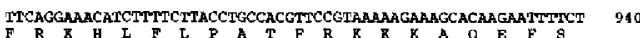

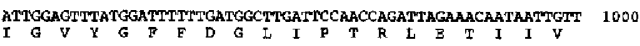
GTTATPTTIGTTGCTTGACGGGTCTCTTCAGIGCCTTACATATPCATCACGTCAMAGAT 1060 AATCCACAATATGCTACTAAGAMTGCTGATTGGGTCATTTGATTGCCGACAGGACTGGT 1120

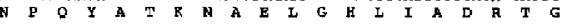

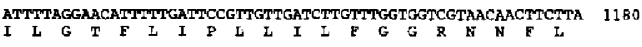
CAATGGCTAACTGGITGGGATTTTGCTACTTPTATCATGTATCACAGATGGATTTCCCET 1240 GTTGATGTTTTATTGATCATCGTTCATGCAATCACTTTTTCCGTGTCTGACAAAGCAACE 1300

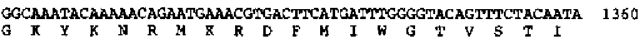

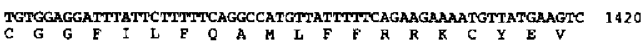

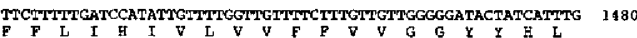

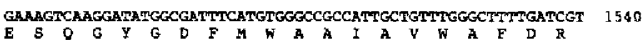
GTCGTCAGATTAGGGAGAATTTCTPTTTCGGTGCCAGAAAAGCAACCGTCTCTATTAAG 1600

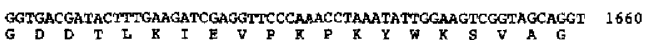

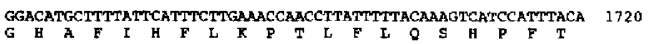

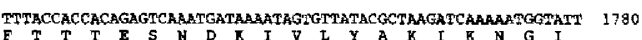

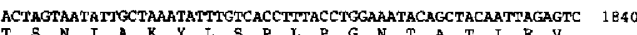

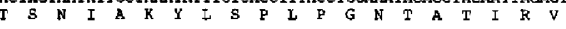
TPAGTGGAAGGTCCGTATGGTGAACCTAGCAGTGCTGGACGTAACTGTAAGAATGTGGTT 1900 TFPGTTGCTGGTGGTAATGGTATTCCAGGTATPTATMCCGAATGTGITGATCTAGCTAAG 1960

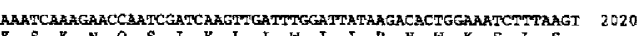

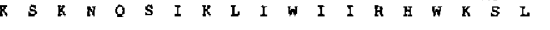

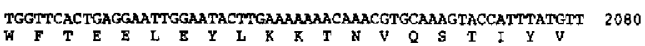
ACTCAACCICAGGATTCCTCAGGATTAGAATGCYTTCAACACGATGTTAGTPTTGAAAAG 2140 AaATCCGACGAAAGGACTCAGTAGAaTCATCTCAATATTCACTCATTMCTAATATTAAG 2200

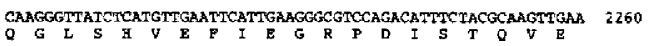

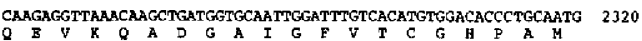
GTTGATGAGTTGAGATTTGCAGTCACCCAGAATTFGAATGTATCAARACACAGAGTGGAS 2380 TAMCACGACACAMTIACAGACATGGGCATAMatcaagtgatacadgatt tgaaaacaaaasc 2440

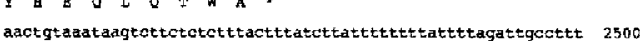
atatattattgatactagtaatacaaatttatacacaact.taaatttttctratttaaă 2560 atatteatgetgtcattetttteccagaaaccgagaaagaatcaaactctatttttattg 2620 tttttgtttcttattcaatttattcgtegtcactatcttcataagtgggtatgtcattaa 2680 gtatttgttgeagttģttgttgttgttettgtettggaggcgtttgatgtccattaccat 2740 tagatrocgt tt taattttettecgtagtggttettegtgcagtacagaagctqgacgag 2800 caactocat tgaaatgettotgtcttetategttctetatct tettecaagcesttgata 2860 ttttgggacaccactcaatgacttctttetctttgacaccatecttgatggataccaaa 2920 tcttttccaccacactgatatgatttccattacttttategtttetgaatcgaagcattt 2980 cccaatatccettettagttgtagattggeqacattcagcaatectgccttgtaat.ggtt 3040 gctctagtgccttcaatctttcccaatcatcatctgatagatct 00 . 280 .

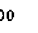

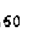

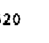
(n)

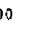
.

20

.

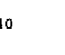

(1)


(a)

C. albicans Cgt $1 p$

s. cerevisiae Cegl

s. pombe pcelp

C. albicans Cgtlp

S. Cerevisiae Cegl
s. pombe Pcelp

C. alblcans Cgtip

S. cerevistae Ce
s. pombe Pcelp

c. albicans cgtlp

S. cerevislae Ceglp

c. albicans Cgt1p

s. cerevislae Cegl

C. albicans cgt 1

s. cerevisiae Ceglp

C. albicans cgtlp

S. cerevisiae Cegl
S. pombe Pcelp

C. albicans cgtla

S. cerevisiae ceg

c. albicans cgtlp

S. cerevisiae ceglp
s. pombe Pcelp

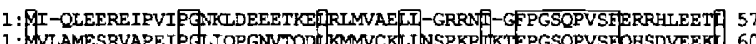

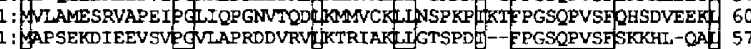

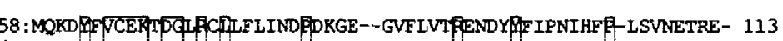

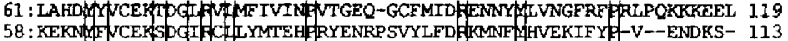

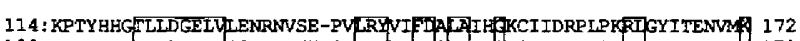

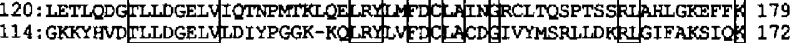

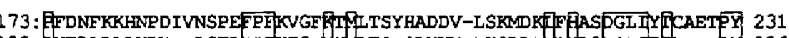

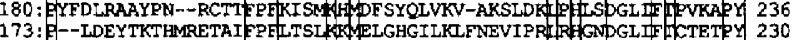

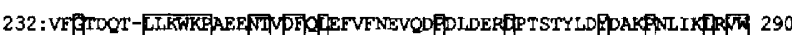

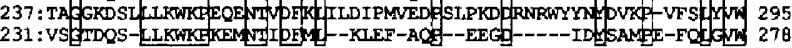

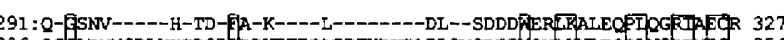

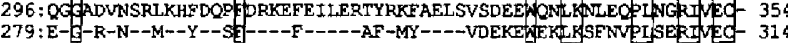

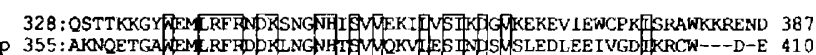

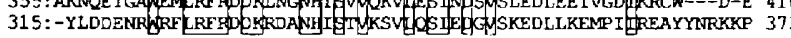

388 : RRQKHFNGVARP-ASVSHEEPLRKKIKTESTNGNGHOTP PRQEQQQQSOQILNDIPTYEDS 446

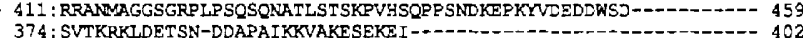

447:DDE 403:---

(b)

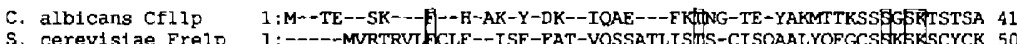

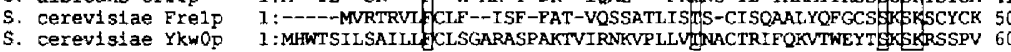

C. albicans Cfllp 42:SKSSKSTGSSNASKSST-NAHG-SNSSTSSTSFSSSKS-GK-GNS-GTSTTETITIPHI 96 s. cerevisiae Fre1p 51:NINWLGSVTACAYENSK-SNKTLDSALMKLA-OQCSSI-KVYTLEDMKNIYLNASNYRA 107 s. Cerevis1ae YkwOp 61:CSYEPAFQSMLYCIYETIDEKGYSNKTL.EKTF ATIKKNCASYSDAIONMTNSEFYOUTWN 120

c. albicans CfIlP 97: DYKKFTPY-KOAYOMS-N-N-NFNLSIMPGSGFTGXWAGI-IA-I-AIF-ANMIKKM-F 146

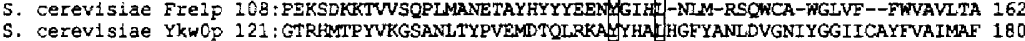

C. albicans CfIlP 147:FSITNNLS-GSISN-LFRKHL--FLPA--TFRK-KKAQEFSIGVYGPPGGLIPTFLETII 199 5. cerev1s1ae Fre1p 163: ATILNILKRVFGKNIM-ANS-VKKSLIYPSVYKOYNERTFYLFRRLPA-NTE TIFGKGLV 218

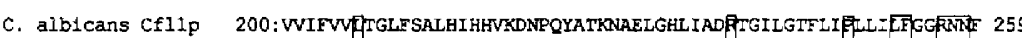
s. cerevisiae Fre1p 219:VLIFVI TILSLSFGHNIKLPHPYDRPFNRRSMAFVSRRADIMAIALF WWYLFGIRNAP 278 S. cerev1siae YkWOP 241: ILGYLVLHTVFLAYGYEYDPENIIFKSRRVQVARYVADESGVLAFAHFEFIVLFRGENV 300

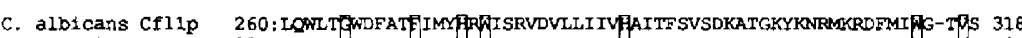
S. cerevisiae FrelP 279:FIPITELSFSTENFYHWMSAYVCFMLAVM SIVMTASGVKRGVFQSLVRKFYFRAGI-VA 33

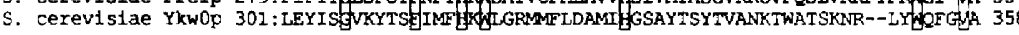

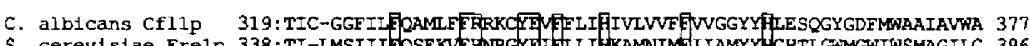

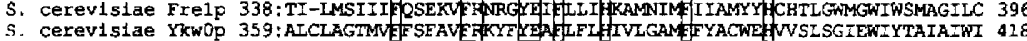

c. albicans Cfllp 378:FPFVRLGRIFFF AAFAATVSIKGPGTLKIEV-PEP-KYWKSVAFGHAFIHFTKF-TL 433

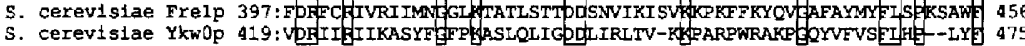

c. alblcans CEli-P 434:--LSSEPFT----FTTTESNDKIV-IYARIKAOIRSNIAKYLSPLPGNTATIRVLVEGPY 486 S. cerevislae FreIp 457: YSFPSHPFIVLSERHRDPANDDQLTMYVAANKGI TRVLLSKVISAPNHTVDCKIFLEGPY 51

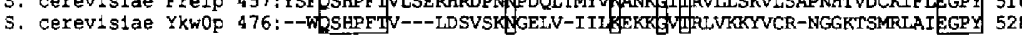

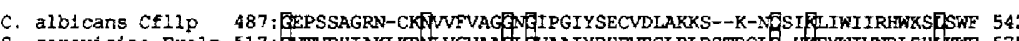
s. cerevisiae Frelp 517: VVTVPHIAKLKR CVGVAA II GVAAIYPHFVECLRLPSTDQID-H价YWIVNDLSHEKWF 57

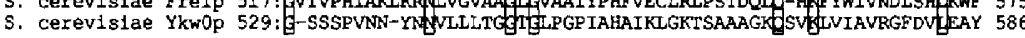

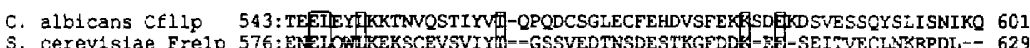

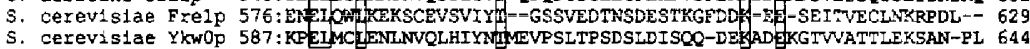

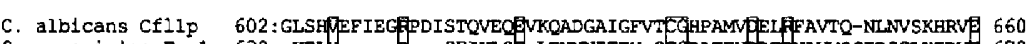
s. cerevisiae Fre1p 630:-KEIM----

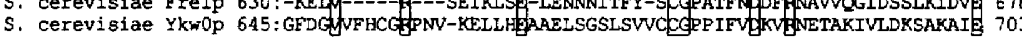

C. alb1 cans CFllp $661:$ YHFELOTFA

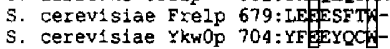

Fig. 3. Comparison of the amino acid sequences of Cgt1p and Cfl1p with homologous proteins in other organisms. (a) The amino acid sequence of $C$ albicans Cgt1p is compared to those of mRNA 5 . guanylyltransferases of $S$. cerevisiae (Ceg1p) and Schiz. pombe (Pce $1 p)$. Identical amino acids among these three proteins are boxed. (b) The amino acid sequence of $C$ albicans $C f \mid p$ is compared to those of $S$. cerevisiae ferric reductase (Fre1p) and Ykw0p, whose function is unknown. Identical amino acids in these three proteins are boxed. Amino acid alignment was carried out using the program in Genetyx version 7.3 .
3). Experiments involving the deletion of the cloned DNA fragment revealed that ORF1 was essential for complementation of the ceg1s null mutation (Fig. 1).
Furthermore, a $2.5 \mathrm{~kb} C l a \mathrm{I}-\mathrm{Cla} \mathrm{I}$ fragment that contained only ORF1 rescued the ceg $1 \Delta$ null mutant in the presence of 5-FOA, even in a single-copy plasmid, whereas a $3.7 \mathrm{~kb}$ 

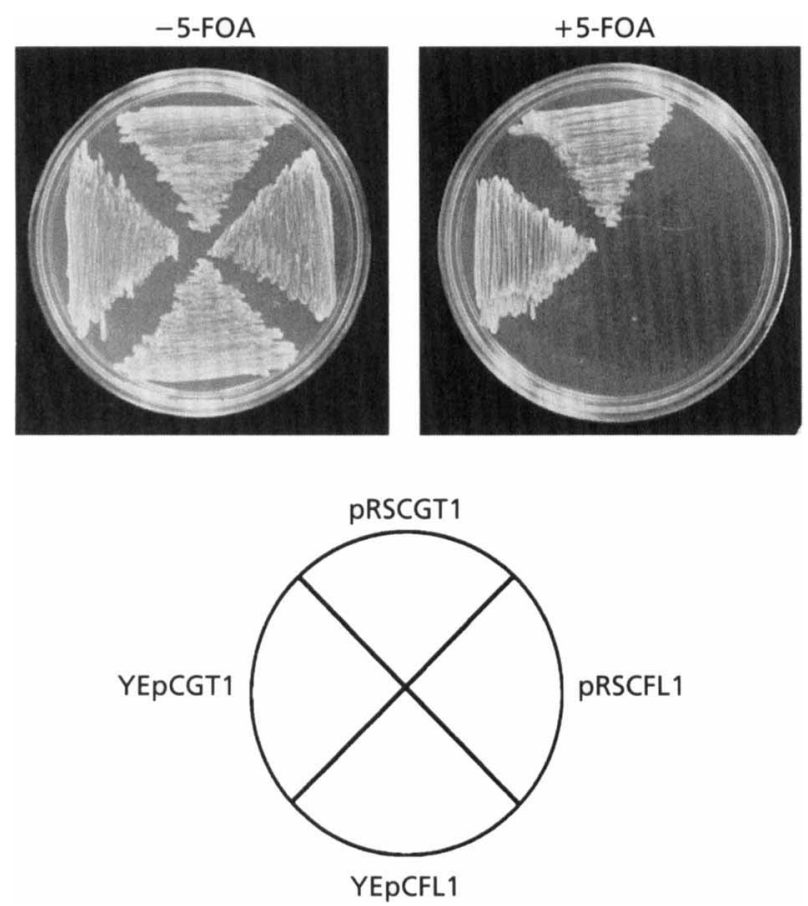

Fig. 4. Complementation of the $S$. cerevisiae cegis null mutation by $C$. albicans CGT1. S. cerevisiae ceg $1 \triangle:: L E U 2$ cells that harboured intact CEG1 in Yep24 were further transfected with YEPCGT1, YEPCFL1, pRSCGT1 or PRSCFL1. The transfectants were then seeded on agar plates with or without 5 -FOA and incubated at $30^{\circ} \mathrm{C}$ for $3 \mathrm{~d}$. For more details, see Methods.

SalI-XbaI fragment that contained only ORF2 did not (Fig. 4). All these results demonstrate that ORF1 encodes $C$. albicans mRNA 5'-guanylyltransferase, and therefore ORF1 was designated CGT1 ( $\underline{C}$. albicans guanylyltransferase gene 1 ).

\section{CGT1 encodes mRNA 5'-guanylyltransferase}

The expected size of Cgt1p deduced from the nucleotide sequence was identical to that of mRNA 5'guanylyltransferase detected by the enzyme-GMP complex formation using crude nuclear extracts of $C$. albicans (data not shown). In S. cerevisiae Ceg1p, Lys ${ }_{70}$ is expected to be the GMP-binding site, and the neighbouring sequence including this lysine, Lys-X-Asp-Gly, in which $\mathrm{X}$ can be any amino acid, is widely conserved in proteins that form a stable nucleotide monophosphate-enzyme complex (Cong \& Shuman, 1993; Fresco \& Buratowski, 1994; Schwer \& Shuman, 1994). Cgt1p also contained the Lys-Thr-Asp-Gly motif starting at position 67 (Fig. 2a).

To further confirm that CGT1 encodes mRNA $5^{\prime}$ guanylyltransferase, we tested whether or not Cgt1p could form a stable complex with the GMP moiety of GTP. To address this possibility, C. albicans Cgt1p and $S$. cerevisiae Ceg1p were expressed in E. coli as fusion proteins with GST, and were purified by affinity column chromatography using glutathione-Sepharose. As shown in Fig. 5, purified GST-Ceg1p and GST-Cgt1p were specifically

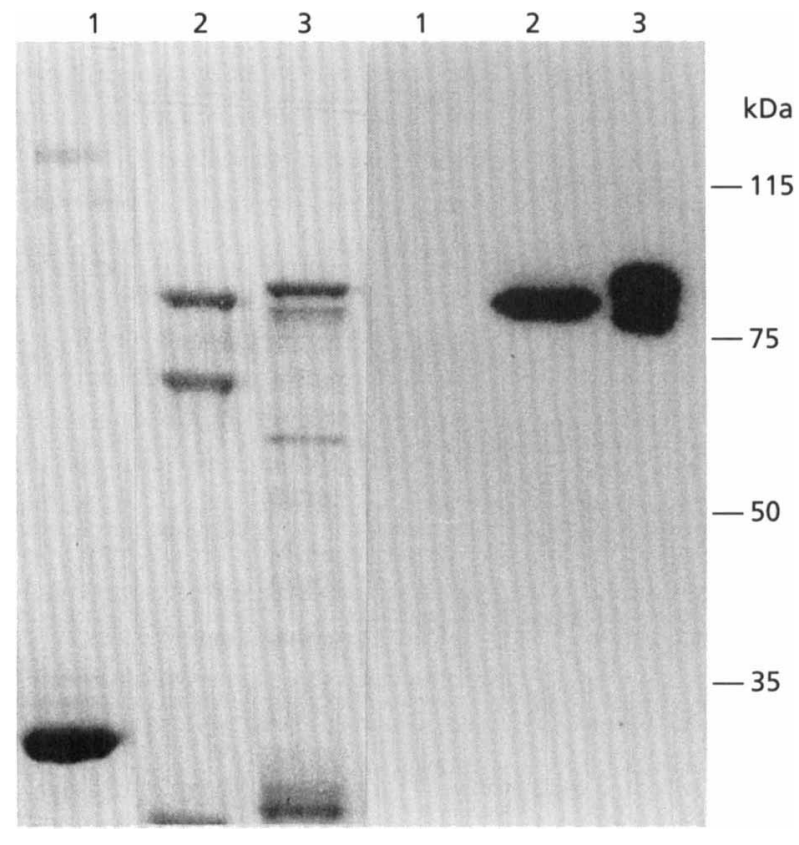

Fig. 5. Expression of Cgt1p in $E$. coli and stable complex formation with the GMP moiety of GTP. S. cerevisiae Ceg1p and C. albicans Cgt1p were expressed in $E$. coli as fusion proteins with GST, and purified by glutathione affinity column chromatography. One microgram of purified GST (lane 1), GST-Ceg1p (lane 2) or GST-Cgt1p (lane 3) was incubated with $\left[\alpha_{-}{ }^{32} \mathrm{P}\right] \mathrm{GTP}$, separated on $10 \%$ SDS-PAGE gels and visualized by staining with Coomassie Brilliant Blue (left panel) or by autoradiography (right panel). Positions of size markers are indicated in kDa. For more details, see Methods.

labelled with ${ }^{32} \mathrm{P}$ when incubated with $\left[\alpha_{-}{ }^{32} \mathrm{P}\right] \mathrm{GTP}$, whereas GST alone was not. The GMP-enzyme complexes were stable, even in the presence of SDS, and were never dissociated through SDS-PAGE (Fig. 5). Furthermore, Cgt1p synthesized GpppG when incubated with GTP and ppG-terminated RNA (Fig. 6). Two bands were labelled with ${ }^{32} \mathrm{P}$ in the purified GS'T-Cgt1p fraction (Fig. 5). Because Coomassie Brilliant Blue staining of the purified Cgtlp also showed two bands whose sizes were identical to those detected by enzyme-GMP complex formation (Fig. 5), the faster-migrating band might be the degraded product of Cgt1p. Binding of Cgt1p to nucleotides was specific to the GMP moiety of GTP, and it had little affinity to ATP, CTP and UTP (data not shown).

Southern blot analysis demonstrated that digestion of $C$. albicans genomic DNA with $B g / \mathrm{II}, E c o \mathrm{RI}$ or $X b a \mathrm{I}$ produced bands of $1.2 \mathrm{~kb}, 0.6$ and $1.2 \mathrm{~kb}$, or $1.9 \mathrm{~kb}$, respectively. This pattern was coincident with the expected size from the restriction map of CGT1, suggesting that CGT1 was a single-copy gene in the $C$. albicans haploid genome (Fig. 7).

\section{Another gene present near the CGT1 locus}

We found another ORF (indicated as ORF2 in Fig. 1) very close to the CGT1 locus in the opposite strand of the CGT1 ORF. ORF2 shared significant sequence homology 


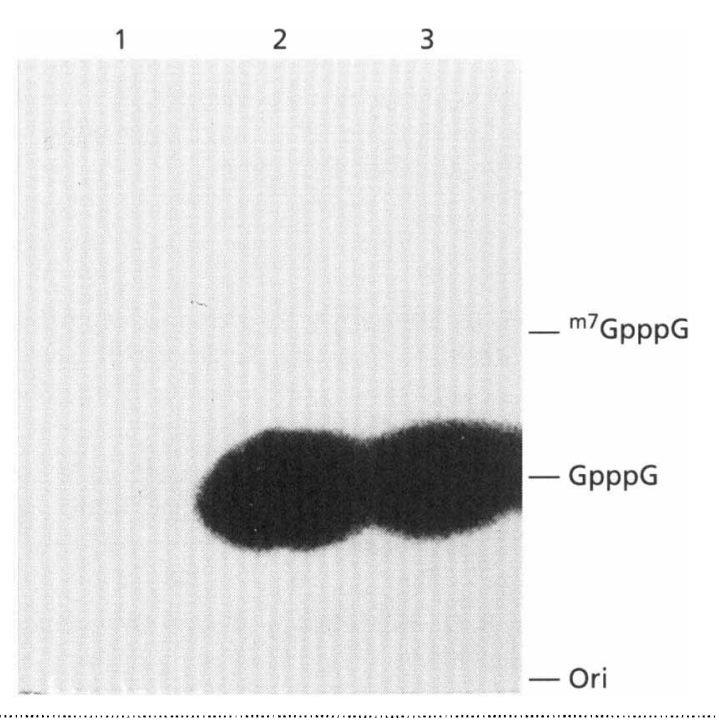

Fig. 6. Cap formation by Cgttp in vitro. One microgram each of purified GST (lane 1), GST-Ceg1p (lane 2) or GST-Cgt1p (lane 3) was incubated with [ $\left.\alpha-{ }^{32} \mathrm{P}\right] \mathrm{GTP}$ and ppG-terminated RNA. After incubation, the RNA was digested with nuclease P1 and calf intestine alkaline phosphatase, and analysed by paper electrophoresis. Positions of GpppG and ${ }^{m 7} \mathrm{GpppG}$ are indicated. For more details, see Methods.

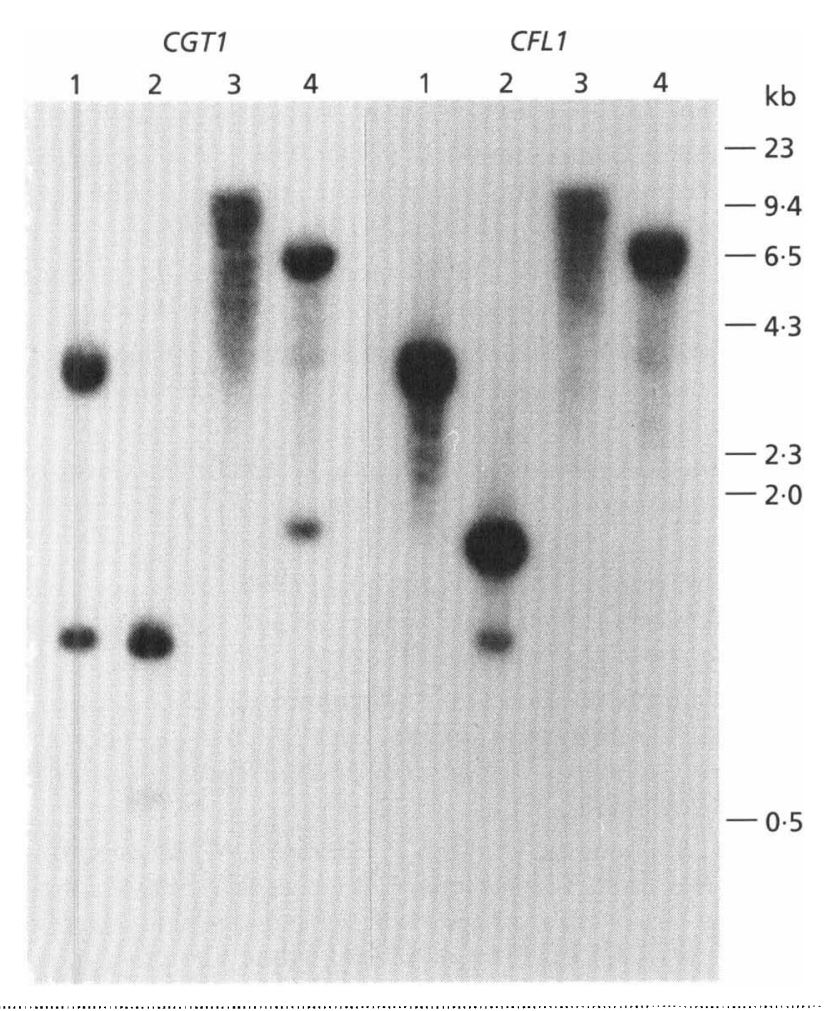

Fig. 7. Southern blotting using $C G T 1$ and $C F L 1$ as probes. $C$. albicans genomic DNA $(25 \mu \mathrm{g})$ was digested with Bglll (lane 1). EcoRI (lane 2), HindIII (lane 3) or Xbal (lane 4), separated on agarose gels, and hybridized with radiolabeljed CGT1 (left panel) or CFL1 (right panel) probe. Positions of size markers are indicated in kb. For more details, see Methods.

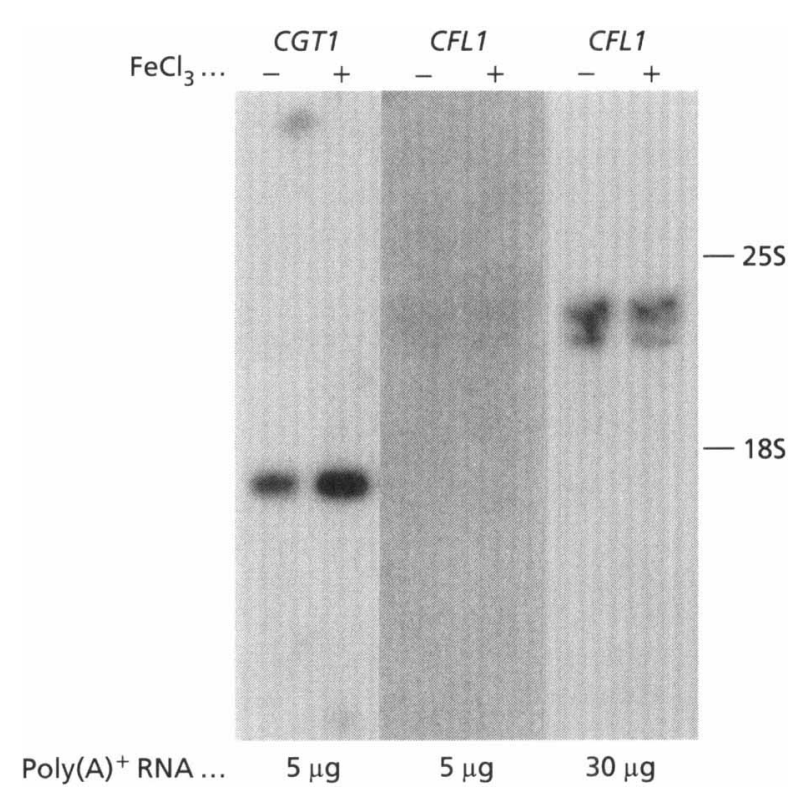

Fig. 8. Northern blotting of CGT1 and CFL1 mRNA. Indicated amounts of poly $(A)^{+}$RNA extracted from $C$. albicans cells that were cultured in MD medium in the absence $(-)$ or presence (+) of $2 \mathrm{mM} \mathrm{FeCl}_{3}$ were separated on agarose gels and hybridized with radiolabelled CGT1 (left panel) or CFL1 (middle and right panels) probes. Positions of 255 and 185 rRNA are indicated. For more details, see Methods.

with $S$. cerevisiate ferric reductase (Fre1p) (Dancis et al, 1992) and a protein of unknown function ( $\mathrm{Ykw} 0 \mathrm{p}$ ) $(28.1 \%$ identity and $69.4 \%$ homology with Fre1p, and $30.7 \%$ identity and $71.5 \%$ homology with $\mathrm{Ykw0p}$, in the entire protein) (Fig. 3b). 'Therefore, ORF2 was designated CFL1 (C. albicans ferric-reductase-like gene 1).

The above facts taise the possibility that $C F L 1$ is the $C$. albicans ferric reductase gene. However, CFL1 in either a single-copy plasmid or a multicopy plasmid did not complement the slow growth of the $S$. cerevisiae fre $1 \Delta$ null mutant in the absence of iron (data not shown) and the ferric reductase activity was not increased when CFL1 was overexpressed in $S$. cerevisiae cells using the alcohol dehydrogenase promoter. Moreover, the CFL1 mRNA level was extremely low compared to that of CGT1, and this level of CFL1 expression was not increased, even when cells were cultured in iron-depleted medium (Fig. 8). Two bands of CFL1 mRNA appeared by Northern blotting (Fig. 8). Since CFL 1 did not contain introns, there seemed to be several transcription start sites in the $C F L 1$ promoter.

\section{DISCUSSION}

We have demonstrated here that CGT1 encodes mRNA $5^{\prime}$-guanylyltransferase, and that the GST-Cgt1p fusion protein expressed in bacterial cells was fully active in forming a stable enzyme GMP complex and in synthesizing the cap structure in vitro. Schiz. pombe PCE1 was also cloned by functional complementation of the $S$. cerevisiae ceg1A null mutation (Shuman et al., 1994). 
Although the expected size of Pcelp (47 kDa) is smaller than that of Ceg1p and Cgt1p (52 kDa) (Shuman et al., 1994), the fact that Ceg1p, Pce1p and Cgtlp share a high degree of sequence homology suggests that mRNA 5'guanylyltransferase is highly conserved in various yeasts. In addition, we did not detect mRNA $5^{\prime}$-triphosphatase activity in the recombinant $\mathrm{Cgt} 1 \mathrm{p}$, indicating that like other yeasts, $C$. albicans CGT1 encodes only mRNA $5^{\prime}$ guanylyltransferase and that mRNA $5^{\prime}$-triphosphatase is encoded by another gene.

Lys $_{70}$ was shown to be the important residue for covalent binding of GMP in S. cerevisiae Ceg1p (Fresco \& Buratowski, 1994; Schwer \& Shuman, 1994), and Lys-XAsp-Gly in which Lys is expected to be the GMP-binding site was widely conserved among the enzymes that form a stable enzyme-nucleotide monophosphate complex (Shuman et al., 1994). Cgt1p also contained the Lys-XAsp-Gly motif near the N-terminus. However, comparison of the $S$. cerevisiae, Scbiz. pombe and $C$. albicans sequences revealed that there were several completely conserved areas (Fig. 3a). Since mRNA 5'-guanylyltransferase should recognize and may bind to $m R N A$, some of these conserved sequences may be responsible for RNA recognition.

Recently, S. cerevisiae ABD1 was identified as the mRNAcap methyltransferase gene (Mao et al., 1995). ABD1 encodes a protein with a molecular mass of $50 \mathrm{kDa}$, and disruption of this gene was lethal in S. cerevisiae cells. Since CEG1 is also an essential gene, it seems likely that every step of mRNA capping is essential for vegetative growth of yeasts, and that yeasts have no isozyme for enzymes synthesizing the mRNA-cap structure.

In $S$. cerevisiae, ORF2 was found adjacent to the CEG1 locus (Shibagaki et al., 1992). As in the S. cerevisiae CEG1 locus, we also identified the CFL1 gene located very close to the $C$. albicans $C G T 1$ locus. Despite its sequence homology with $S$. cerevisiae FRE1, the results that $C F L 1$ expression was not induced by iron deprivation and that $C F L 1$ did not complement the fre $1 \Delta$ null mutation of $S$. cerevisiae suggest that CFL1 is functionally distinct from S. cerevisiae FRE1. Although the physiological function of $S$. cerevisiae $Y K W 0$ remains to be established, CFL1 may be functionally related to $S$. cerevisiae $Y K W O$ rather than FRE1. However, we cannot tule out the possibility that the failure of CFL1 to substitute for $S$. cerevisiae FRE1 was the consequence of the different usage of the CUG codon between C. albicans and S. cerevisiae : it is decoded as serine and not leucine in $C$. albicans (Santos \& Tuite, 1995). In fact, there is one CTG in the CFL1 ORF (at position 548 in Fig. 2b). In S. cerevisiae, ferric reductase is coupled to the ferrous transporter to convert biologically inaccessible ferric $\left(\mathrm{Fe}^{3+}\right)$ ion into the ferrous $\left(\mathrm{Fe}^{2+}\right)$ state (Dancis et al., 1990), and at least two ferric reductases are present and encoded by the distinct genes FRE1 and FRE2 (Georgatsou \& Alexandraki, 1994). We do not know if $C$. albicans incorporates iron by a similar mechanism to $S$. cerevisiae, but identification of the ferric reductase genes in $C$. albicans would answer this question.

\section{ACKNOWLEDGEMENTS}

We thank Mr Yabe for his technical assistance. This work was supported in part by grants from Human Science and from the Ministry of Education and Science, Japan.

\section{REFERENCES}

Botstein, D., Falco, S. C., Stewart, S. E., Brennan, M., Scherer, S., Stinchcomb, D. T., Struhl, K. \& Davis, R. W. (1979). Sterile host yeasts (SHY): a eukaryotic system of biological containment for recombinant DNA experiments. Gene 8, 17-24.

Cong, P. \& Shuman, S. (1993). Covalent catalysis in nucleotidyl transfer: a KTDG motif essential for enzyme-GMP complex formation by mRNA capping enzyme is conserved at the active sites of RNA and DNA ligases. J Biol Chem 268, 7256-7260.

Dancis, A., Klausner, R. D., Hinnebusch, A. G. \& Barriocanal, J. G. (1990). Genetic evidence that fertic reductase is requircd for iron uptake in Saccharomyces cerevisiae. Mol Cell Biol 10, 2294-2301.

Dancis, A., Roman, D. G., Anderson, G. J., Hinnebusch, A. G. \& Klausner, R. D. (1992). Ferric reductase of Saccharomyces cerevisiae: molecular characterization, role in iron uptake, and transcriptional control by iron. Proc Natl Acad Sci USA 89, 3869-3873.

Edery, I. \& Sonenberg, N. (1985). Cap-dependent RNA splicing in a HeLa nuclear extract. Proc Natl Acad Sci USA 82, 7590-7594.

Filipowicz, W. (1978). Functions of the $5^{\prime}$-terminal $m^{7} \mathrm{G}$ cap in eukaryotic mRNA. FEBS Lett 96, 1-11.

Fresco, L. D. \& Buratowski, S. (1994). Active site of the mRNAcapping enzyme guanylyltransferase from Saccbaromyces cerevisiae: similarity to the nucleotidyl attachment motif of DNA and RNA ligases. Proc Natl Acad Sci US.A 91, 6624-6628.

Furuichi, Y., LaFiandra, A. \& Shatkin, A. J. (1977). 5'-Terminal structure and mRNA stability. Nature 266, 235-239.

Georgatsou, E. \& Alexandraki, D. (1994). Two distinctly regulated genes are required for ferric reduction, the first step of iron uptake in Saccbaromyces cerevisiae. Mol Cell Biol 14, 3065-3073.

Hamm, J. \& Mattaj, I. W. (1990). Monomethylated cap structures facilitate RNA export from the nucleus. Cell 63, 109-118.

Inoue, K., Ohno, M., Sakamoto, H. \& Shimura, Y. (1989). Effect of the cap structure on pre-mRNA splicing in Xenopus oocyte nuclei. Genes Dev 3, 1472-1479.

Ito, H., Fukuda, Y., Murata, K. \& Kimura, A. (1983). Transformation of intact yeast cells treated with alkali cations. $J$ Bacteriol 153, 163-168.

Itoh, N., Mizumoto, K. \& Kaziro, Y. (1984). Messenger RNA guanylyltransferase from Saccharomyces cerevisiae. I. Purification and subunit structure. J Biol Chem 259, 13923-13929.

Itoh, N., Yamada, H., Kaziro, Y. \& Mizumoto, K. (1987). Messenger RNA guanylyltransferase from Saccharomyces cerevisiae: large scale purification, subunit functions, and subcellular localization. $J$ Biol Chem 262, 1989-1995.

Kasahara, S., Yamada, H., Mio, T., Shiratori, Y., Miyamoto, C. Yabe, T., Nakajima, T., Ichishima, E. \& Furuichi, Y. (1994). Cloning of the Sacharomyces cerevisiae gene whose overexpression overcomes the effects of HM- 1 killer toxin, which inhibits $\beta$-glucan synthesis. J Bacteriol 176, 1488-1499.

Konarska, M. M., Padgett, R. A. \& Sharp, P. A. (1984). Recognition of cap structure in splicing in vitro of $\mathrm{mRNA}$ precursors. $C_{e} / l 38$, $731-736$

Krainer, A. R., Maniatis, T., Ruskin, B. \& Green, M. R. (1984). Normal and mutant $\beta$-globin pre-mRNA are faithfully and efficiently spliced in vitro. Cell 36, 993-1005. 
Kuribayashi-Ohta, K., Tamatsukuri, S., Hikata, M., Miyamoto, C. \& Furuichi, Y. (1993). Application of oligo(dT) ${ }_{30}$-latex for rapid purification of poly $(A)^{+} m R N A$ and for hybrid subtraction with the in situ reverse transcribed cDNA. Biochim Biopbys Acta 1156, 204-212.

Lundblad, v. (1989). Saccharomyces cerevisiae. In Current Protocols in Molecular Biology, pp. 13.11.1 13.11.5. Edited by F. M. Ausubel, R. Brent, R. E. Kingston, D. D. Moore, J. E. Seidman, J. A. Smith \& K. Struhl. New York: Wiley Interscience.

Mao, X., Schwer, B. \& Shuman, S. (1995). Yeast mRNA cap methyltransferase is a 50-kilodalton protein encoded by an essential gene. Mol Coll Biol 15, 4167-4174.

Mizumoto, K. \& Kaziro, Y. (1987). Messenger RNA capping enzymes from eukaryotic cells. Prog Nucleic Acid Res Mol Biol 34, $1-28$.

Mizumoto, K. \& Lipmann, F. (1979). Transmethylation and transguanylylation in 5 -RNA capping system isolated from rat liver nuclei. Proc Natl Acad Sci US A 76, 4961-4965.

Murthy, K. G. K., Park, P. \& Manley, J. L. (1991). A nuclear micrococcal-sensitive, ATP-dependent exoribonuclease degrades uncapped but not capped RNA substrates. Nucleic Acids Res 19, 2685-2692.

Niles, E. G., Condit, R. C., Caro, P., Davidson, K., Matusick, L. \& Seto, J. (1986). Nucleotide sequence and generic map of the 16-kb vaccinia virus HindIII D fragment. Virology 153, 96-112.

Odds, F. C. (1987). Candida infection: an overview. Crit Rey Microbiol 15, 1-5.

Ohno, M., Sakamoto, H. \& Shimura, Y. (1987). Preferential excision of the 5 proximal intron from mRNA precursors with two introns as mediated by the cap structure. Proc Natl Acad Sci USA 84, 5187-5191.

Patzelt, E., Thalmann, E., Hartmuth, K., Blaas, D. \& Kuechler, E. (1987). Assembly of pre-mRNA splicing complex is cap dependent. Nucleic Acids Res 15, 1387-1399.

Pena, L., Yanez, R. J., Revilla, Y., Vinuela, E. \& Salas, M. L. (1993). African swine fever virus guanylyltransferase. Virology 193, 319-328.

Sambrook, J., Fritsch, E. F. \& Maniatis, T. (1989). Molecular Cloning : A Laboratory Manual, 2nd edn. Cold Spring Harbor, NY: Cold Spring Harbor Laboratory.

Santos, M. A. S. \& Tuite, M. F. (1995). The CUG codon is decoded in vivo as serine and not leucine in Candida albicans. Nucleic Acids Res 23, $1481-1486$.
Scherer, S. \& Magee, P. T. (1990). Genetics of Candida albicans. Microbiol Rev 54, 226-241.

Schwer, B. \& Shuman, S. (1994). Mutational analysis of yeast mRNA capping enzyme. Proc Natl Acad Sci US A 91, $4328-4332$.

Seliger, L. S., Zheng, K. \& Shatkin, A. J. (1987). Complete nucleotide sequence of reovirus $\mathrm{I}, 2$ gene and deduced amino acid sequence of viral mRNA guanylyltransferase. I Biol Chem 262, 16289-16293.

Shatkin, A. J. (1976). Capping of eukaryotic mRNAs. Cell 9 , 645-653.

Shatkin, A. J. (1985). mRNA cap binding proteins : essential factors for initiation of translation. Cell 40, 223-224.

Shibagaki, Y., Itoh, N., Yamada, H., Nagata, S. \& Mizumoto, K. (1992). mRNA capping enzyme: isolation and characterization of the gene encoding mRNA guanylyltransferase subunit from Saccharomyces cerevisiat. J Biol Chem 267, 9521-9528.

Shimotohno, K., Kodama, Y., Hashimoto, J. \& Miura, K. I. (1977). Importance of $5^{\prime}$-terminal blocking structure to stabilized mRNA in eukaryotic protein synthesis. Proc Natl Acad Sci USA 74, $2734-2738$.

Shuman, S. (1995). Capping enzyme in eukaryotic mRNA synthesis. Prog Nucleic Acid Res Mol Biol 50, 101-129.

Shuman, S., Liu, Y. \& Schwer, B. (1994). Covalent catalysis in nucleotidyl transfer reactions: essential motifs in Sacharomyces cerevisiae RNA capping enzyme are conserved in Scbizosaccharomyces pombe and viral capping enzymes and among polynucleotide ligases. Proc Natl Acad Sci USA 91, 12046-12050.

Smith, D. B. \& Johnson, K. S. (1988). Single-step purification of polypeptides expressed in Escherichia coli as fusions with glutathione $S$-transferase. Gene 67, 31-40.

Struhl, K., Stinchcomb, D. T., Scherer, S. \& Davis, R. W. (1979). High-frequency transformation of yeast: autonomous replication of hybrid DNA molecules. Proc Natl Acad Sci USA 76, 1035-1039.

Upton, C., Stuart, D. \& McFadden, G. (1991). Identification and DNA sequence of the large subunit of the capping enzyme from Shope fibroma virus. Virology 183, 773-777.

Yagi, Y., Mizumoto, K. \& Kaziro, Y. (1983). Association of an RNA 5 -triphosphatase activity with RNA guanylyltransferase partially purified from rat liver nuclei. EMBO J 2, 611-615.

Received 29 December 1995; revised 19 March 1996; accepted 1 April 1996. 